\title{
ESTIMATIVA DA POROSIDADE DRENÁVEL DE UM SOLO DE VÁRZEA A PARTIR DA TENSÃO DA ÁGUA NO SOLO
}

\author{
J.E. QUEIROZ1; D.E. CRUCIANI2; P.L.LIBARDI ${ }^{3,4}$ \\ ${ }_{2}^{1}$ Depto. de Eng. Florestal-UFPB/Campus VII, C.P. 64, CEP: 58.700-970, Patos, PB \\ ${ }_{3}$ Depto. de Engenharia Rural-ESALQ/USP, C.P. 9, CEP: 13418-900 - Piracicaba, SP \\ ${ }^{3}$ Depto. de Fisica e Meteorologia-ESALQ/USP, C.P. 9, CEP: 13418-900 - Piracicaba, SP \\ 4 Bolsista do CNPq
}

RESUMO: Um modo alternativo para estimativa da porosidade drenável em função da tensão da água no solo a partir da equação de VAN GENUCHTEN para a curva de retenção, é apresentado e testado para dados obtidos de um solo de várzea ( Gleissolo Eutrofico, textura argilosa). Curvas de retenção de água foram elaboradas com amostras de solo com estrutura indeformada. Na analise dos resultados verificou-se que o maior componente de erro foi devido a causas aleatorias, representando cerca de 12 vezes o erro sistemático. Os indices estatísticos utilizados para verificar 0 grau de ajuste dos dados experimentais à relaçāo funcional apresentada entre porosidade drenável e tensão de água no solo, mostraram que esta relação pode ser utilizada para estimativa da porosidade drenável, a partir do conhecimento dos parâmetros empíricos da equação de VAN GENUCHTEN.

Descritores: Porosidade drenável; Curva de retenção; Parâmetros empíricos

\section{ASSESSMENT OF DRAINABLE POROSITY OF A HOLM SOIL FROM SOIL-WATER TENSION}

SUMMARY: An alternative way to assess the drainable porosity as a function of soil-water tension, from the soilwater retention curve (Van Genuchten's model), is presented for data of a clayey holm gley soil of the county of Piracicaba, SP, Brazil. Soil-water retention curves were determined using undisturbed soil samples. The analysis of the results has shown that the data variability was mainly due to random causes. Statistical indexes used to verify the degree of fitting of the experimental data to the proposed model, have shown that the model can be used to estimate the drainable porosity from the empirical parameters of the Van Genuchten model.

Key words: Drainable porostity; Retention curve; Empirical parameters.

\section{INTRODUÇÃO}

A porosidade drenável constitui um parâmetro de grande importância no dimensionamento de sistemas de drenagem, como também em estudos de modelos matemáticos para predição da altura do lençol freático no espaço entre linhas de drenos paralelos.

Vários autores (PIZARRO, 1978; BELTRAN, 1986; MILLAR, 1988; entre outros) consideram a porosidade drenável como um parâmetro constante e equivalente à macroporosidade do solo, podendo ser determinado pela diferença entre os conteúdos de água no solo na saturação e na capacidade de campo. OLIVEIRA (1968) cita vários autores (PEELE, 1949; UHLAND, 1949; VAN DOREN, 1949; GROHMANN, 1960) que utilizaram como valor limite de separação entre a macro e micro porosidade uma tensão de 60 centímetros de coluna d'água (c.c.a.) ou $6 \mathrm{kPa}$, a qual corresponde aos poros de diâmetro da ordem de $0.05 \mathrm{~cm}$.

Considerar a porosidade drenável como uma constante significa assumir uma condição drenagem capaz de levar o solo do ponto de saturação até a "capacidade de campo", onde seria cessado o processo de drenagem interna (equilíbrio estático). Esta condição pode não ser a mais adequada do ponto de vista de dimensionamento de sistemas de drenagem agrícola, onde o critério de drenagem é estabelecido em termos da dinâmica do lençol freático, conforme as exigências das culturas a serem exploradas. Para determinadas situações pode ser mais conveniente a utilização de uma tensão menor, com a qual se determina a respectiva porosidade livre de água, a qual 
corresponde à porosidade drenável para a tensão considerada.

A porosidade drenável como uma função da tensâo de água ou posição da capa freática tem sido reconhecida por vários pesquisadores (LUTHIN \& WORSTELL, 1957; TAYLOR, 1959; DUKE, 1972; SKAGGS, 1976; BHATTACHARYA, 1979; CRUCIANI, 1983; PENDEY et al., 1992; GUPTA, 1994), porém continua sendo ignorada em muitas pesquisas de drenagem.

Estre trabalho tem como objetivo apresentar e testar um modo altenativo para estimar a porosidade drenável de um solo em função da tensão de água no solo, a partir dos parâmetros obtidos da equação de VAN GENUCHTEN (1980).

\section{MATERIAL E MÉTODOS}

Numa área experimental de $15 \times 90 \mathrm{~m}$, localizada no campo experimental de drenagem da ESALQ/USP, foram coletadas aleatoriamente 8 amostras de solo com estrutura indeformada, nas profundidades de 30 e $50 \mathrm{~cm}$, totalizando 16 amostras. $O$ solo apresenta textura argilosa, sendo classificado como G2 Gleissolo Eutrófico (VIDALTORRADO \& SPAROVEK, 1993).

A extração das amostras foi feita com anéis volumétricos de bordas cortantes de $3 \mathrm{~cm}$ de altura e $4.7 \mathrm{~cm}$ de diâmetro, aproximadamente (BLAKE, 1986; KLUTE, 1986). No dia anterior à coleta das amostras ocorreu uma chuva que proporcionou o umedecimento do solo, facilitando a introdução manual dos anéis.

No Laboratório de Solos do Departamento de Engenharia Rural da ESALQ/USP, as amostras foram devidamente preparadas e saturadas por um período de aproximadamente 10 dias. Após o processo de saturação, as amostras foram pesadas, sendo posteriormente colocadas em uma mesa de tensão onde foram submetidas às sucções de 1,2 , 4 e 6 kPa (LEAMER \& SHAW, 1941; OLIVEIRA, 1968). Para completar a curva de retenção de água, foram utilizadas câmaras de pressão de Richards, onde as amostras foram submetidas às pressões de $10,40,80,600$ e $1500 \mathrm{kPa}$ (RICHARDS, 1954).

Para converter a umidade à base de massa (U) em umidade à base de volume (q), utilizou-se a seguinte relação:

$$
\theta=\mathbf{U} \cdot \frac{\rho}{\rho_{\mathbf{a}}}
$$

onde $\rho$ é a densidade do solo e $P_{a}$ a densidade da água no solo considerada igual a $1 \mathrm{~g}^{\mathrm{cm}} \mathrm{cm}^{-3}$.

Para verificar a variabilidade dos dados, para cada tensão, determinou-se o valor médio de umidade, o coeficiente de variação e o desviopadrão. Aplicou-se o teste " $t$ " para igualdade de duas médias através da equação (2) (FONSECA \& MARTINS, 1993):

$$
t=\frac{\left(\bar{\theta}_{1}-\bar{\theta}_{2}\right)-d}{S_{c} \cdot \sqrt{\frac{N_{1}+N_{2}}{N_{1} \cdot N_{2}}}}
$$

onde,

$\bar{\theta}_{1}$ - umidade média para cada tensão e profundidade $\mathrm{z}_{1}=30 \mathrm{~cm}$;

$\bar{\theta}_{2}$ - umidade média para cada tensão e profundidade $\mathrm{Z}_{2}=60 \mathrm{~cm}$;

d-diferença admitida entre as médias $\bar{\theta}_{1}$ e $\bar{\theta}_{2}$;

$N_{1}$ - número de amostras para determinação de $\bar{\theta}_{1}$;

$\mathrm{N}_{2}$-número de amostras para determinação de $\bar{\theta}_{2}$; $\mathrm{S}_{\mathrm{c}}$ - desvio-padrão comum às amostras obtidas em $z_{1} \in z_{2}$.

O valor de $\mathrm{S}_{\mathrm{c}}$ é dado pela seguinte expressão:

$$
S_{c}=\sqrt{\frac{\left(N_{1}-1\right) \cdot S_{1}^{2}+\left(N_{2}-1\right) \cdot S_{2}^{2}}{N_{1}+N_{2}-2}}
$$

onde,

$\mathrm{s}_{1}{ }^{2}$ - variância dos valores de umidade com média $\overline{\boldsymbol{\theta}}$;

$\mathrm{S}_{2}{ }^{2}$ - variância dos valores de umidade com média $\bar{\theta}$

A equação de VAN GENUCHTEN (1980), expressa conforme a equação (4), permite estimar a umidade do solo para qualquer tensão $h_{i}$.

$$
\theta_{i}=\theta_{r}+\frac{\left(\theta_{s}-\theta_{r}\right)}{\left[1+\left(\alpha \cdot h_{i}\right)^{n}\right]^{m}}
$$


onde,

$q_{i}$ - umidade a base de volume $\left(\mathrm{cm}^{3} / \mathrm{cm}^{3}\right)$ correspondente ao i-esimo valor absoluto de hi (cm);

$q_{8}$ - umidade de saturaçăo $\left(\mathrm{cm}^{3} / \mathrm{cm}^{3}\right)$;

$\mathrm{q}_{\mathrm{r}}$ - umidade residual $\left(\mathrm{cm}^{3} / \mathrm{cm}^{3}\right)$;

$h_{i}$ - valor absoluto de tensão de umidade em $\mathrm{cm}$ de água (c.c.a.);

m, n - partmetros adimensionais da equação;

a - parámetro com dimensão igual ao inverso da tensão $\left(\mathrm{cm}^{-1}\right)$.

$O$ parâmetro a tem sido referido como o inverso da tensão ou pressão correspondente a entrada de ar (VAN GENUCHTEN, 1980).

Por definição, a porosidade livre de água $\left(m_{\imath}\right)$ pode ser expressa como:

$$
\mu_{\mathrm{a}}=\eta-\theta_{1}=\theta_{\mathrm{s}}-\theta
$$

onde $h$ E a porosidade total do solo.

Considerando a porosidade livre de água como sendo igual a porosidade drenável ou efetiva para uma dada tensão $h_{\mathfrak{j}}$, isto $\epsilon$, $m_{a}=m\left(h_{i}\right)$, das equações (4) $e(5)$ deduz-se que:

$$
\mu\left(h_{1}\right)=\left(\theta_{s}-\theta_{r}\right) \cdot\left\{1-\frac{1}{\left[1+\left(\alpha \cdot h_{\imath}\right)^{n}\right]^{m}}\right\}
$$

Tomando como fator de escala a porosidade do solo na sua capacidade máxima de retenção $\left(m_{0}\right)$, a equação (6) pode ser expressa como:

$\mu^{*}\left(h_{2}\right)=\frac{\left(\theta_{s}-\theta_{r}\right)}{\mu_{0}} \cdot\left\{1-\frac{1}{\left[1+\left(\alpha \cdot h_{l}\right)^{n}\right]^{m}}\right\}$

onde,

$m^{*}\left(h_{\mathfrak{i}}\right)$-porosidade drenável escalonada como uma função da tensão $h_{i}$;

$\mathbf{m}_{0}$-porosidade do solo correspondente

a diferença entre $\mathrm{q}_{\mathrm{s}}$ e a umidade na "capacidade de campo" $\left(\mathrm{q}_{\mathrm{cc}}\right)$.
Para obter os valores escalonados de porosidade drenável como uma função dos valores medidos de umidade, $m^{*}\left(q_{1}\right)$, com o objetivo de comparar os resultados experimentais com os estimados pela equação (7), utilizou-se a seguinte expressão:

$$
\mu *\left(\theta_{1}\right)=\frac{\theta_{s}-\theta_{1}}{\mu_{0}}
$$

Assumindo o teor de água na "capacidade de campo" como sendo igual ao teor de agua correspondente ao ponto de inflexão da curva de retenģão $\left(q_{\text {inf }}\right)$, o valor de $m_{0}$ pode ser obtido por:

$$
\mu_{0}=\left(\theta_{s}-\theta_{\text {inf }}\right)
$$

As equaçōes (7) e (8) estão sujeitas às seguintes condições de contorno:

$$
\begin{aligned}
& \text { (1) } q_{i}=q_{1} p_{h_{i}}=0 \text { P } m *\left(q_{i}\right)=m *\left(h_{i}\right)=0 \\
& \text { (ii) } q_{i}=q_{\text {inf }} p_{h_{i}}=h_{\text {inf }}>m *\left(q_{i}\right)=m *\left(h_{i}\right)=1
\end{aligned}
$$

A partir da equação (4), explicitando $h$, obtendo a derivada segunda $c$ igualando a zero, pode-se deduzir a expressão (10), a qual permite determinar a umidade no ponto de inflexão( $\left(\mathcal{q}_{\mathrm{inf}}\right)$.

$$
\theta_{\text {inf }}=\left(\theta_{s}-\theta_{r}\right) \cdot\left(\frac{m}{m+1}\right)^{m}+\theta_{r}
$$

Como $\mathrm{m}^{*}\left(\mathrm{~h}_{\mathrm{i}}\right)$ varia entre 0 e 1 , os valores de $h_{i}$ que satisfazem esta condição devem ser tomados no intervalo de umidade entre o ponto de inflexão e o ponto de saturação $\left(q_{\text {inf }}\right.$ $\left.<q<q_{s}\right)$. Para qualquer valor de $m^{*}\left(h_{\mathfrak{j}}\right)$, a porosidade drenável $m\left(h_{\mathfrak{j}}\right)$ pode ser obtida pelo produto $m^{*}\left(h_{\mathfrak{j}}\right) \cdot m_{0}$.

Para verificar o grau de ajuste dos valores estimados de porosidade drenável em relação aos resultados experimentais, foram utilizados o coeficiente de determinaçäo $\left(R^{2}\right)$ e o indice " $D$ " proposto por WILLMOTT (1981), sendo este último calculado pela equação (11). 


$$
D=1-\frac{\sum_{i=1}^{N}\left[\hat{\mu}^{*}\left(h_{1}\right)-\mu^{*}\left(\theta_{1}\right)\right]^{2}}{\sum_{1=1}^{N}\left[\left|\hat{\mu}^{*}\left(h_{1}\right)-\bar{\mu}^{*}\left(\theta_{1}\right)\right|+\left|\mu^{*}\left(\theta_{1}\right)-\bar{\mu}^{*}\left(\theta_{1}\right)\right|\right]^{2}}
$$

onde,

$$
\hat{\mu}^{*}\left(h_{i}\right) \text { - valores estimados de }
$$
porosidade drenável (escalonados) em função de $h_{\mathbf{i}}$;

$$
\mu^{*}\left(\theta_{1}\right) \text { - valores de porosidade }
$$
drenável (escalonados), calculados em função dos valores medidos de umidade $\mathrm{q}_{\mathrm{i}}$;

$$
\bar{\mu}^{*}\left(\theta_{1}\right) \text { - média dos valores de }
$$

$$
\mu^{*}\left(\theta_{1}\right)
$$

Para calcular os erros sistemático $\left(\mathrm{E}_{\mathrm{S}}\right)$, não sistemático $\left(\mathrm{E}_{\mathrm{ns}}\right)$ e o erro médio absoluto $\left(\mathrm{EM}_{\mathrm{a}}\right)$, foram utilizadas as equações (12), (13) e (14), respectivamente (WILLMOTT, 1981):

$$
\begin{aligned}
& E_{s}=\left\{\frac{1}{N} \cdot \sum_{i=1}^{N}\left[\mu^{*}\left(r_{1}\right)-\mu^{*}\left(\theta_{1}\right)\right]^{2}\right\}^{1 / 2} \\
& E_{n s}=\left\{\frac{1}{N} \cdot \sum_{i=1}^{N}\left[\mu^{*}\left(r_{t}\right)-\mu^{*}\left(h_{1}\right)\right]^{2}\right\}^{1 / 2} \\
& \operatorname{EM}_{\mathrm{a}}=\frac{1}{N} \cdot \sum_{1=1}^{N}\left|\mu^{*}\left(h_{1}\right)-\mu^{*}\left(\theta_{1}\right)\right|
\end{aligned}
$$

Os valores de $m^{*}\left(r_{j}\right)$ foram obtidos a partir da regressão linear entre $m^{*}\left(h_{i}\right)$ e $m *\left(q_{i}\right)$.

\section{RESULTADOS E DISCUSSÃO}

Para uma mesma tensão de água, considerando as 8 repetições de cada profundidade de amostragem, os valores obtidos de umidade volumétrica $\left(\mathrm{cm}^{3} / \mathrm{cm}^{3}\right)$ apresentaram uma pequena variação. Para profundidade de $30 \mathrm{~cm}$, os valores mínimos e máximos de desvio-padrão e coeficiente de variação foram $0.024 \mathrm{~cm}^{3} / \mathrm{cm}^{3}, 0.053 \mathrm{~cm}^{3} / \mathrm{cm}^{3}$, $5.48 \%$ e $8.57 \%$, respectivamente. Os maiores valores obtidos de c.v. são inferiores aos menores valores observados por outros autores ao estudarem a variabilidade espacial e temporal da umidade de um solo hidromórfico cinzento, textura média, desenvolvido a partir de sedimentos aluvionais (LIBARDI et al., 1992).

Os valores médios de umidade das amostras obtidas na profundidade de $50 \mathrm{~cm}$, para uma mesma tensão, foram ligeiramente superiores aos obtidos na profundidade de $30 \mathrm{~cm}$ (Figura 1 ). Este fato pode está associado ao aumento da porcentagem de argila com a profundidade, acarretando um aumento na capacidade de retenção de água pelo solo. Para amostra conjunta obteve-se uma curva de retenção intermediária, conforme mostra a Figura 1.

Aplicando o teste " $t$ " para igualdade das médias de umidade, utilizando-se as equações (2) e (3), ao nível de significância de $1 \%$, verifica-se que as médias de umidade podem ser consideradas como provenientes de uma mesma população. Portanto, considerando-se a amostra conjunta (profundidades de 30 e $50 \mathrm{~cm}$ ), os valores mínimos e máximos de desvio-padrão e c.v. foram $\mathbf{0 . 0 3 1 5}$ $\mathrm{cm}^{3} / \mathrm{cm}^{3}, 0.0389 \mathrm{~cm}^{3} / \mathrm{cm}^{3}, 5,50 \%$ e $7,26 \%$, respectivamente.

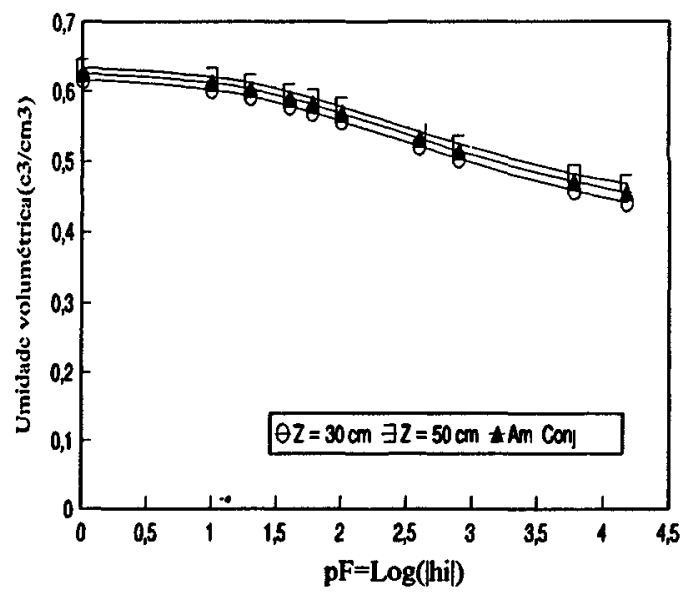

Figura 1 - Curvas de retenção para amostras obtidas nas profundidades de 30 e $50 \mathrm{~cm}$ e para amostra conjunta. 
Com as tensões e os valores médios de umidade da amostra conjunta, foram obtidos os parâmetros da equação de VAN GENUCHTEN (equação 4) através da utilização do programa computacional desenvolvido por DOURADO NETO et al (1990). O programa $\mathrm{cm}^{3}$ realizou um total de 604 iterações e forneceu os seguintes resultados: $q_{s}=0.626 \mathrm{~cm}^{3} / \mathrm{cm}^{3}, q_{r}=0.357 \mathrm{~cm}^{3} /$ $\mathrm{cm}, \mathrm{q}_{\text {inf }}=0.548 \mathrm{~cm}^{3} / \mathrm{cm}^{3}, \mathrm{a}=0.0299 \mathrm{~cm}^{-1}$, $\mathrm{m}=0.1859$ e $\mathrm{n}=0.8909$. O coeficiente de ajuste dos dados ao modelo $\left(R^{2}\right)$ foi de 0.997 . Com estes resultados pode-se escrever a seguinte relação funcional entre umidade $e$ tensão:

$\theta_{\imath}=0.357+\frac{0.269}{\left[1+\left(0.0299 . h_{\imath}\right)^{08909}\right]^{01859}}$

Para porosidade drenável, expressa na forma da equação (6), obteve-se a seguinte relação funcional:

$\mu\left(h_{i}\right)=0.269 .\left\{1-\frac{1}{\left[1+\left(0.0299 . h_{i}\right)^{08909}\right]^{01859}}\right\}$

A equação (6a) permite estimar a porosidade drenável para qualquer tensão de água. A Figura 2 mostra a relação entre a porosidade drenável, expressa em \%, e a tensão de água, expressa em c.c.a. e em termos absolutos. No ponto de saturação $\left(h_{i}=0\right), m(0)=0$. Para uma tensão máxima de 15000 c.c.a. obteve-se um valor médio $m(15000)=17.12 \%$. Para esta tensão, em solos da série "Luiz de Queiroz" e "Sertãozinho", para profundidade de $45 \mathrm{~cm}$, SCARDUA (1972) obteve os valores de 17.86 e $23.92 \%$, respectivamente. Observa-se ainda na Figura 2 que na faixa de pequenas tensões, uma pequena variação de tensão, proporciona uma grande variação na porosidade drenável. Um aumento de tensão de $\mathbf{4 0}$ para 100 c.c.a., por exemplo, proporcionou uma variação de $60 \%$, aproximadamente, na porosidade drenável, enquanto um aumento de tensão de 6000 para 15000 c.c.a., proporcionou uma variação de apenas 10 $\%$, aproximadamente.

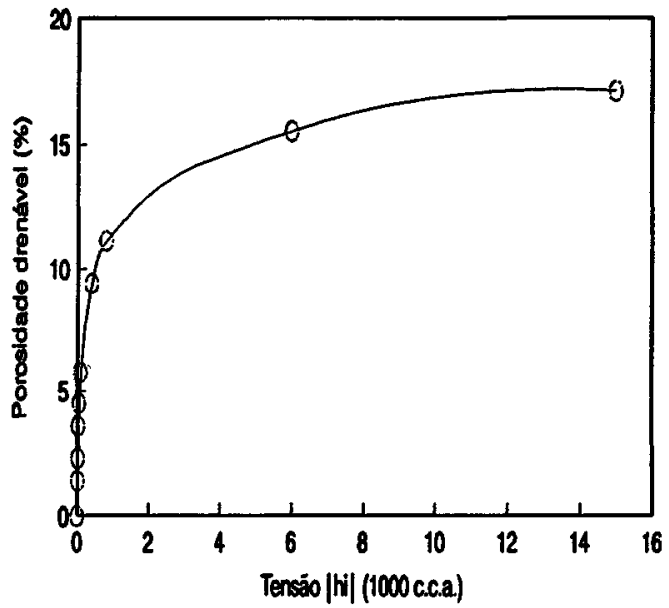

Figura 2 - Porosidade drenável em função da tensão da água no solo (amostra conjunta).

Com a umidade correspondente ao ponto de inflexão $\left(q_{\text {inf }}\right)$, da equação (4a) obteve-se $h_{i}$ aproximadamente igual a $218 \mathrm{~cm}$. Esta seria teoricamente a tensão necessária para levar a umidade do solo do ponto de saturação até a "capaciade de campo", correspondendo a mais de 3 vezes o valor limite de separação entre a macro e microporosidade citado por OLIVEIRA (1968).

Em termos de drenagem interna no perfil do solo, a umidade de $0.548 \mathrm{~cm}^{3} / \mathrm{cm}^{3}$ seria atingida na camada superfícial do solo quando 0 lençol freático permanecesse em equilíbrio na profundidade de aproximadamente 2.2 metros. Entre este valor de umidade e o ponto de saturação, qualquer valor de $h_{i}(0$ a $218 \mathrm{~cm})$ pode $s e r$ considerado para estimativa da porosidade drenável escalonada, expressa na forma da equação (7).

A porosidade drenável correspondente a "capacidade de campo" $\left(\mathrm{m}_{\mathrm{o}}\right)$, obtida pela diferença entre a umidade de saturação e a umidade do ponto de inflexão, resultou num valor de $0.078 \mathrm{~cm}^{3} / \mathrm{cm}^{3}$. Com este valor de $m_{o}$ e introduzindo os parâmetros da equação de VAN GENUCHTEN (1980) na equação (7), obteve-se a seguinte relação funcional para porosidade drenável escalonada:

$\mu^{*}\left(h_{t}\right)=3.4487 .\left\{1-\frac{1}{\left[1+\left(0.0299 . h_{i}\right)^{08909}\right]^{0.1859}}\right\}$ 
A equação (7a) permite estimar a porosidade drenável admensional para qualquer valor de $h_{\mathbf{i}}$. Os valores de $m^{*}\left(h_{\mathfrak{i}}\right)$ estimados através da equação (7a) e os valores de $m^{*}\left(q_{i}\right)$ determinados em função dos dados de umidade do solo (equação 8), plotados em função da tensão de água resulta a Figura 3. Plotando-se os valores de $\mathrm{m}^{*}\left(\mathrm{~h}_{\mathbf{i}}\right)$ versus $\mathrm{m}^{*}\left(\mathrm{q}_{\mathbf{i}}\right)$ obtém-se a Figura 4. Observa-se nesta Figura uma concentração dos pontos em relação a reta $1 / 1$, resultando a alto valor do coeficiente de determinação $\left(R^{2}=0,992\right)$. Observa-se não haver uma tendência no sentido de caracterizar erros sistemáticos na estimativa de $\mathrm{m}^{*}\left(\mathrm{~h}_{\mathfrak{j}}\right)$. Calculandose o erro sistemático através da equação (12) obtevese um valor de $2,316.10^{-3}$, enquanto o erro não sistemático obtido por meio da equação (13) foi de $2,668 \cdot 10^{-2}$. Portanto, o erro não sistemático é cerca de 12 vezes o erro sistemático, indicando que a maior parte do erro pode ser atribuída a causas aleatórias. $O$ erro médio absoluto, calculado através da equação (14), foi de $0,0185 \mathrm{~cm}^{3} / \mathrm{cm}^{3}$.

Utilizando-se a equação (11) obteve-se um índice " $D$ " igual a 0,993 . A pequena diferença entre o $R^{2}$ e o ídice " $D$ ", neste caso, está relacionada com o pequeno valor do erro sistemático, porém em situações onde este componente de erro é considerável, as diferenças podem ser bem maiores.

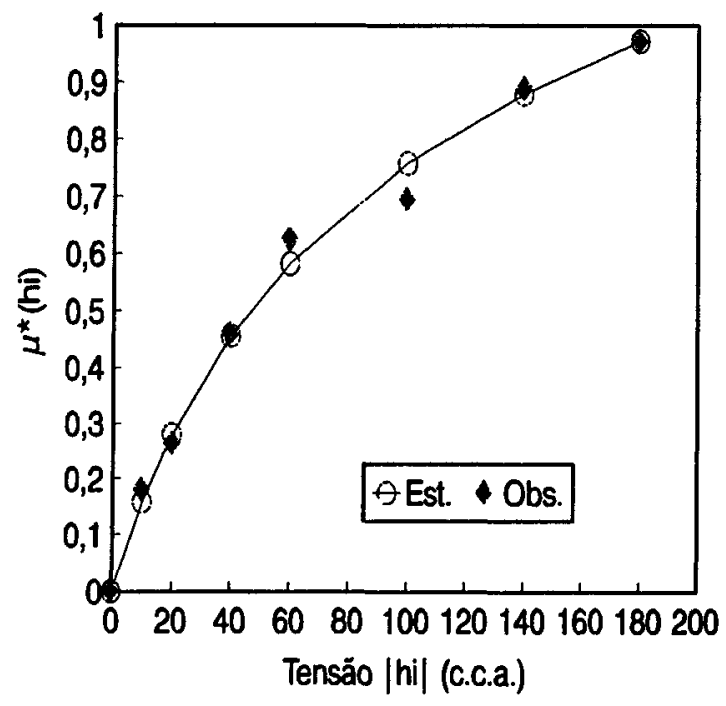

Figura 3 - Valores estimados e observados de porosidade drenável (escalonados) em função da tensão de água no solo.

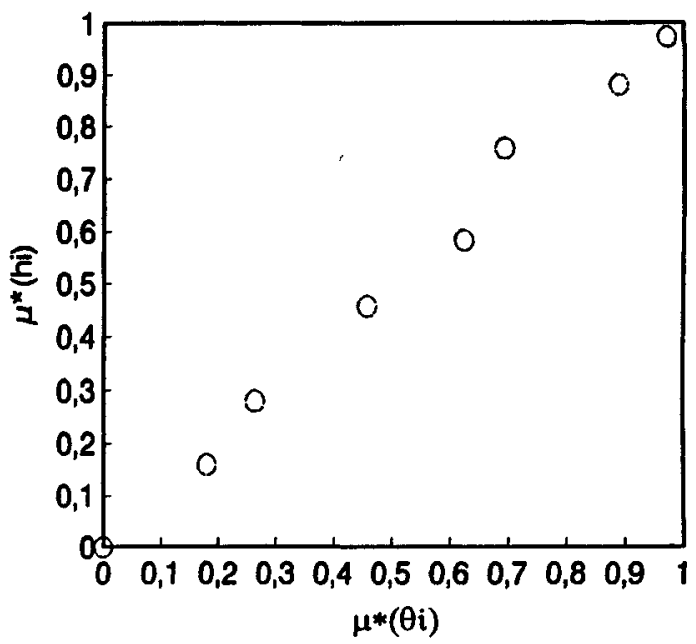

Figura 4 - Relação entre valores estimados e observados de porosidade drenável (escalonados).

\section{CONCLUSÕES}

Pela análise dos resultados apresentados neste trabalho verifica-se que a porosidade drenável pode ser estimada em função da tensão de água no solo, a partir do conhecimento dos parâmetros da equação de Van Genuchten. Trata-se de uma relação funcional que pode ser bastante útil em estudos de drenagem em condições de regime variável, onde a porosidade drenável constitui um importante parâmetro a ser considerado.

\section{REFERÊNCIAS BIBLIOGRÁFICAS}

BELTRAN, J.M. Drenaje agrtcola. Madrid: Iryda, 1986 239 .

BHATTACHARYA, A.K.; BROUGHTON, R.S. Variable drainable porosity in drainage design. Journal of the Irrigation and Drainage Division, New York, v.105, n.1, p.71-85, 1979.

BLAKE, A. Bulk density. In: Black, C.A., ed. Methods of soil analysis. I. Physical and mineralogical methods. Madison: American Society of Agronomy, 1986. p.363-75.

CRUCIANI, D.E. A drenagem na agricultura. 2.ed. São Paulo: Nobel, 1983. 337p. 
DOURADO NETO, D.; LIER, Q. J.V.; BOTREL, T.A.; LIBARDI, P.L. Programa para confecção da curva de retenção da água no solo utilizando o modelo de Genuchten. Engenharia. Rural, Piracicaba, v.1, n.2, p.92-102, 1990.

DUKE, H.R. Capillary properties of soils: influence upon specific yield. Transactions of the ASAE, St. Joseph, v.15, n.4, p.688-91, 1972.

FONSECA, J.S.; MARTINS, G.A. Curso de estatistica. São Paulo: Atlas, 1993. 317p.

GUPTA, S.K. Unsteady drainage with variable drainage porosity. Journal of the Irrigation and Drainage Engineering, New York, v.120, n.4, p.703-15, 1994.

KLUTE, A. Water retention: a laboratory methods. In: Black, C.A., ed. Methods of soil analysis. I. Physical and mineralogical methods. Madison: American Society of Agronomy, Inc., Soil Science Society of America, Inc. Publisher. 1986. p.635-662.

LEAMER, R.W.; SHAW, B. A simple apparatus for measuring noncapillary porosity on an extensive scale. Jonrnal of the American Society of Agronomy, washington, v.33, n.11, p.1003-8, 1941.

LIBARDI, P.L.; MANFRON, P.A.; MORAES, S.O.; TUON, R.L. Variabilidade espacial e temporal da umidade de um solo hidromórfico do R.G. do Sul. In: CONGRESSO BRASILEIRO DE ENGENHARIA AGRiCOLA, 21., Santa Maria, 1992. Anais. Santa Maria: Sociedade Brasileira de Engenharia Agrícola, 1992. p.431-41.

LUTHIN, J.N.; WORSTELL, R.V. The falling water table in tile drainage: a laboratory study. Soll Science Society of America Proceedings, Maidson, v.21, p.580-84, 1957.

MILlAR, A.A. Drenagem de terras agrtcolas: bases agronômicas. São Paulo:Editerra, 1988. 306p.

OLIVEIRA, L.B. Determinação da macro e microporosidade pela "mesa de tensão" em amostras de solo com estrutura indeformada. Pesquisa Agropecuaria Brasileira, Brasilia, v.3, p.197-200, 1968.
PENDEY, R.S.; BHATTACHARYA, A.K.; SINGH, O.P.; GUPTA, S.K. Drawdown solution with variable drainable porosity. Journal of the Irrigation and Drainage Division, New York, v.118, n.3, p.382-96, 1992.

PIZARRO, F. Drenaje agricola y recuperacion de suelos salinos. Madrid:Agrícola Espanhola, 1978. 525p.

RICHARDS, L.A. Diagnosis and improvement of salineardalalinesolis Washington: United States Salinity Laboratory Staff, 1954. 160p. (Agriculture Handbook, 60).

SCARDUA, R. Porosidade livre de água de dois solos do Município de Piracicaba, SP. Piracicaba, 1972. 83p. Dissertação (M.S.) - Escola Superior de Agricultura "Luiz de Queiroz", Universidade de São Paulo.

SKAGGS, R.W. Saturated and unsaturated flow to parallel drains. Journal of the Irrigation and Drainage Division, New York, v.102, n.2, p. 221-38, 1976.

TAYLOR, G.S. Drainage porosity evaluation from outflow measurement and its use in drawdown equations. Soll Science, Baltimore, v.90, n.6, p.338-43, 1960.

VAN GENUCHTEN, M. Th. A closed-form equation for predicting the hydraulic conductivity of unsaturated soils. Soul Science Society of America Journal, Madtson, v. 44, p.892-8, 1980.

VIDAL-TORRADO, P.; SPAROVEK, G. Mapa pedol6gico detalhado do campus "Luiz de Queiroz" (Escala 1:10000). Piracicaba: Universidade de São Paulo, 1993 (Não publicado).

WILLMOTT, C.J. On the validation models. Physical Geography, Madison, v.2, p.184-94, 1981.

Recebido para publicação em 16.03.95

Aceito para publicação em 10.04 .95 